Jaurnal of Environmental Science, Computer Science and Engineering \& Technology

An International Peer Review E-3 Journal of Sciences and Technology

Available online at www.jecet.org

Section B: Computer Science

Research Article

\title{
Adaptive Propagation of Signal for Service Quality in Wireless Networks using Smart Antenna System (SAS)
}

\author{
Ayeni, A. Gabriel and Kabari, L. Giok
}

Post Graduate Unit (Ph.D), Department of Computer Science, Faculty of Natural and Applied Sciences, Post Graduate School, Rivers State University of Education, Port Harcourt, Nigeria.

Received: 21 November 2019; Revised: 04 December 2019; Accepted: 10 December 2019

\begin{abstract}
A smart antenna system allows combination of antenna arrays through signal processing capacity for transmission and adaptive reception. Smart antennas normally complement the signal quality of wireless by enhancing capacity through increased radio frequency. This paper focused on the quantitative analysis and performance experiment of smart antennas for signal quality in wireless network. Virtual wireless network was created for signal propagation and simulated in MATLAB using adaptive array. Performance analysis was examined, by measuring the signal intensity against noise ratio and throughput. The simulation results showed that adaptive beam array produces weight vectors to optimize radiation pattern at steady flow of the amplitude by minimizing the interference.
\end{abstract}

Keywords: Adaptive array, Propagation, Smart antennas, Signal quality, Wireless network

\section{INTRODUCTION}

Smart antennas can synchronize signals from multiple antenna elements with processing capability to improve radiation and reception in response to the environment ${ }^{1}$. Whenever connection flow migrates on the network, it is expected to use the available bandwidth of the link ${ }^{2}$. Abundance of networks 
may bring about diminishing quality of service received by user, therefore it is necessary to focus on the quality of service (QoS) that is being provided to users ${ }^{3}$.

Individual transmission of data packet through computer network, especially by internet users can affect the service quality; hence, a study on network routing is germane to improvement. According to previous studies, network routing is somehow elusive because the topology may change steadily and accessible routing report is inherently indefinite ${ }^{4}$.

Smart antennas are solution artifact to increasing the spectral effectiveness and improving the system performance in mobile communication equipment ${ }^{5}$. In communication context, radio frequency (RF) signal is propagated from the transmitter to exterior and, in vice versa, to the receiver from the exterior ${ }^{6}$.

However, antennas have been undermined among the components in mobile communication and network systems. Whereas, the modality for energy distribution to and fro space has a great influence on the efficient use of spectrum, and the service quality provided. Antenna is considered to be smart when it can recognize and track the signal of a particular mobile device while suppressing interferences. The advent of radio technology digital signal processors, had given to room to curiosity of applications and suitability for signal quality. Hence, optimizing signal radiation and reception in wireless network through experimental propagation of adaptive and smart antennas is the focus of this paper.

\section{RELATED LITERATURE}

Nowadays, network is being trusted for every human undertaken through modern communication and Internet of Things (IoT), which allow communications between man and machine or machine to machine; for the purpose of building control and factory automation. Fuzzy logic model for managing the quality of service in mobile and wireless networks; with respect to technical limitations such as limited bandwidth, power instability, mobility, dynamic topology, network scalability and multi-hop routing which are detrimental to the quality of service had been provided ${ }^{7}$.

Routing and Wireless Network: Routing is the process of getting information packets where they need to go. Routing is an astounding complicated task, and there are a number of diverse algorithms used to find the shortest route between two points ${ }^{8}$. Distance between two nodes communicating with each other at a particular time is calculated; as important factor in transmitting a packet directly which depends on the distance between the nodes ${ }^{9}$. Routing plays a significant role for providing a well delivering qualitative service in networks ${ }^{10}$.

There are many bandwidth optimization and quality of service techniques that have been used to deliver mobile broadband with high performance; these techniques require high level investment and commitment from those core service providers ${ }^{11}$. Designing a suitable congestion control mechanism which will ascertain rational allocation of available bandwidth and to schedule video frames to peers is important ${ }^{12}$. Congestion control algorithm must be stable and adaptive with a wide range of scalability parameters on network ${ }^{2}$.

Peer to peer congestion control and avoidance method, which will cushion the effect of peer to peer traffic on conventional internet gateway ${ }^{12}$. Congestion occurs during transmission of the data from source to sink, control is a means to improve the performance when demand for the finite transmission capacity exceeds the supply ${ }^{9}$. Router operates like a special computer that executes a software program which determines optimal route for the data to reach its destination. 


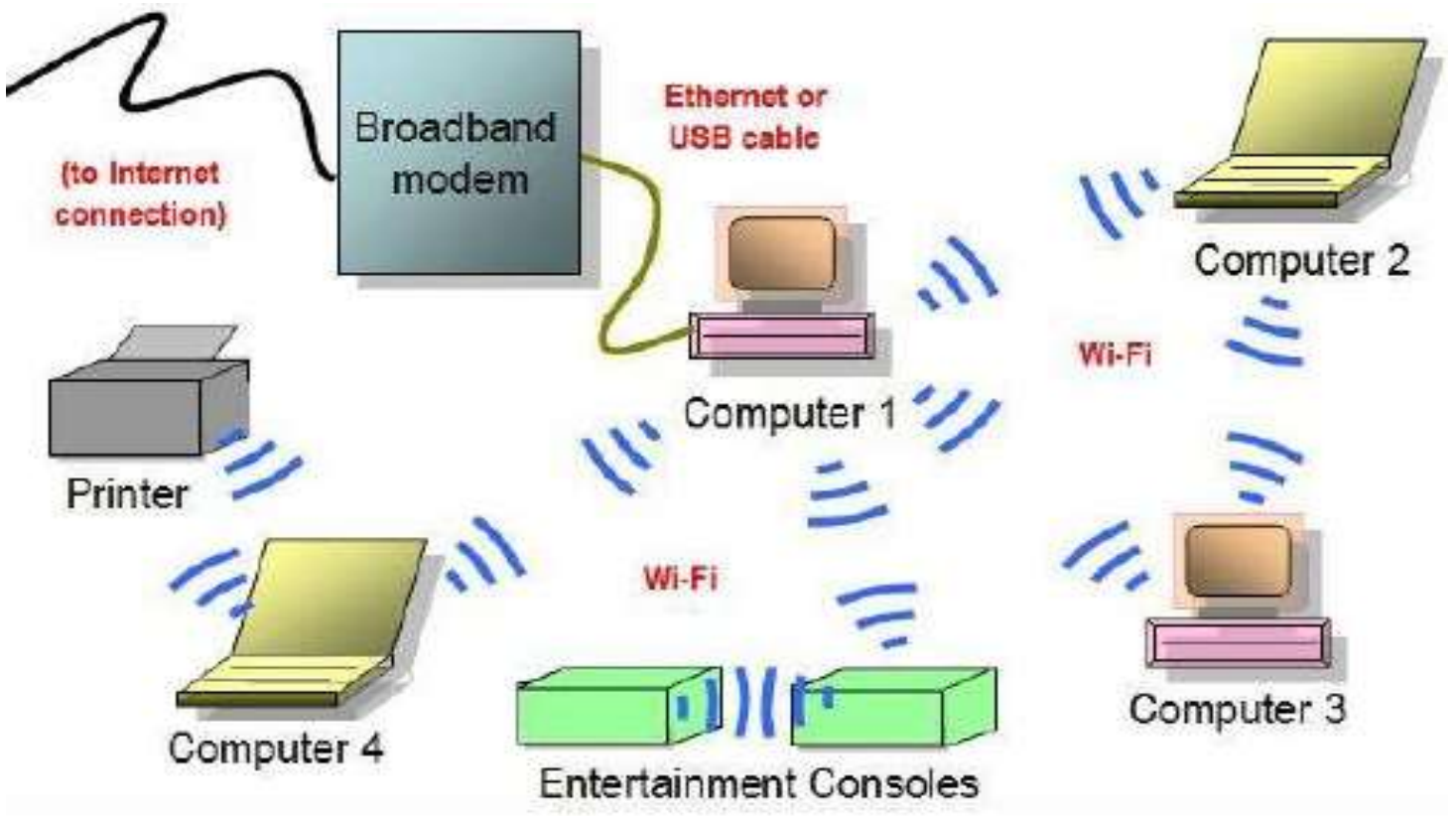

Fig. 1: Wireless Network Infrastructure ${ }^{1 .}$

Smart Antenna and Propagation: Smart Antenna System (SAS) is a subsystem that comprised of several antennas, due to signal processing, in order to improve wireless network performance ${ }^{13}$. The overall performance depends largely on the routing algorithms and capacity ${ }^{14}$.

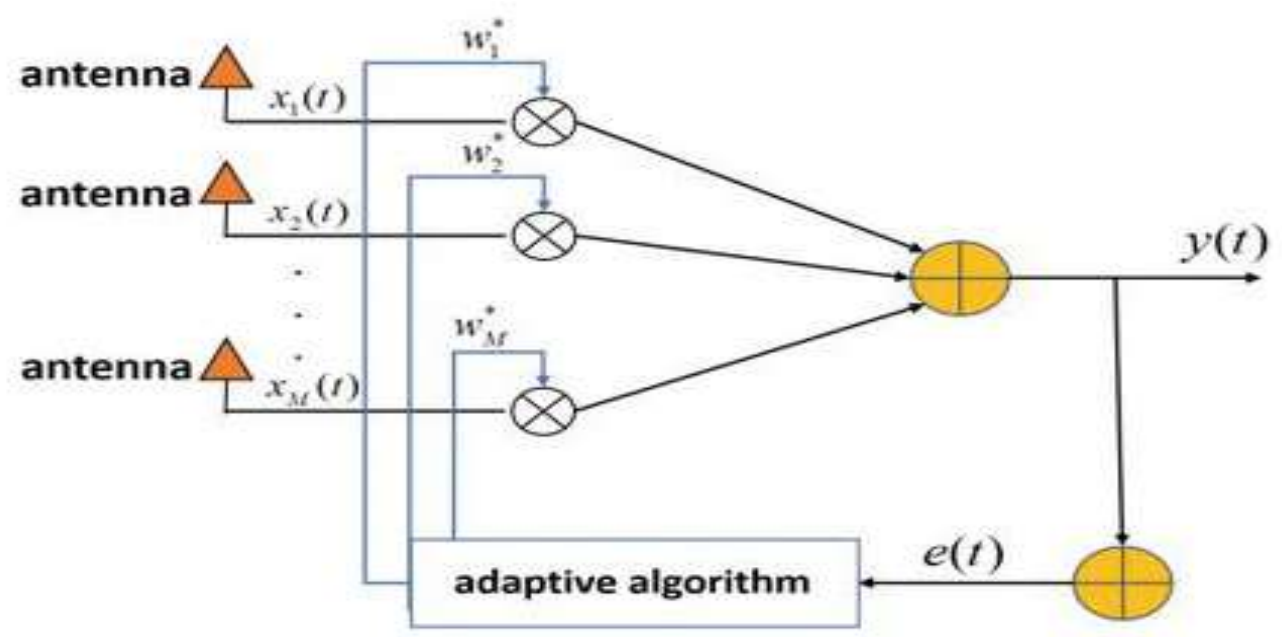

Fig. 2: Adaptive Propagation for Smart Antennas ${ }^{15}$

\section{METHODOLOGY}


This work adopt quantitative and experimental design by simulating a virtual wireless network to demonstrate the efficiency of propagation algorithm for radiation; due to the required capital expenditure to test-run network transmission by setting up wireless network in a laboratory scenario. Simulation in MATLAB was implored in order to demonstrate the efficacy of adaptive algorithm for pattern radiation and signal propagation from multiple antenna elements.

Inputs $\mathrm{x} 1(\mathrm{t}), \square, \mathrm{xM}(\mathrm{t})$ are multiplied by elements of a weight vector $\mathrm{W}=[\mathrm{w} 1, \mathrm{w} 2, \ldots . \mathrm{wM}]$ that varies according to an adaptive algorithm; $y(t)$ is the output, while $e(t)$ denotes the error; discrete time $t$. Adaptive array constitute infinite number of patterns. The simulation parameters were ten (10) antenna elements, two-point-four (2.4) GHz frequency and initial weight of 0.001 each.

\section{EXPERIMENT AND RESULTS}

Performance analysis was examined, by measuring the signal intensity against noise ratio and throughput. The simulation results showed that adaptive beam array produces weight vectors to optimize radiation pattern at steady flow of the amplitude by minimizing the interference.

The Signal to Noise Ratio (SNR) was increased by seventy-five percent (75\%) which has really minimized the channel interference, while the Throughput was increased by ninety-five percent $(95 \%)$ as a remarkable packet delivery, as shown in the figures below.
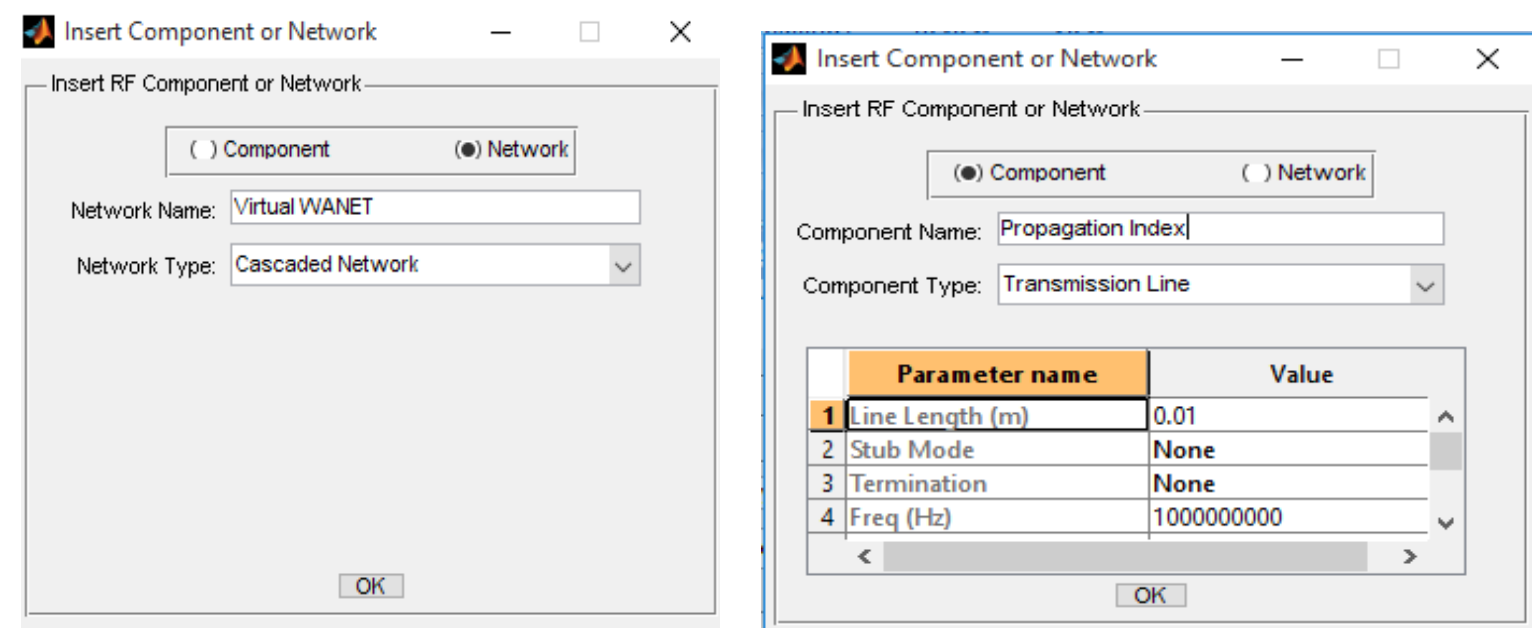

Fig. 3: MATLAB Dialog for Virtual Network and the Component 


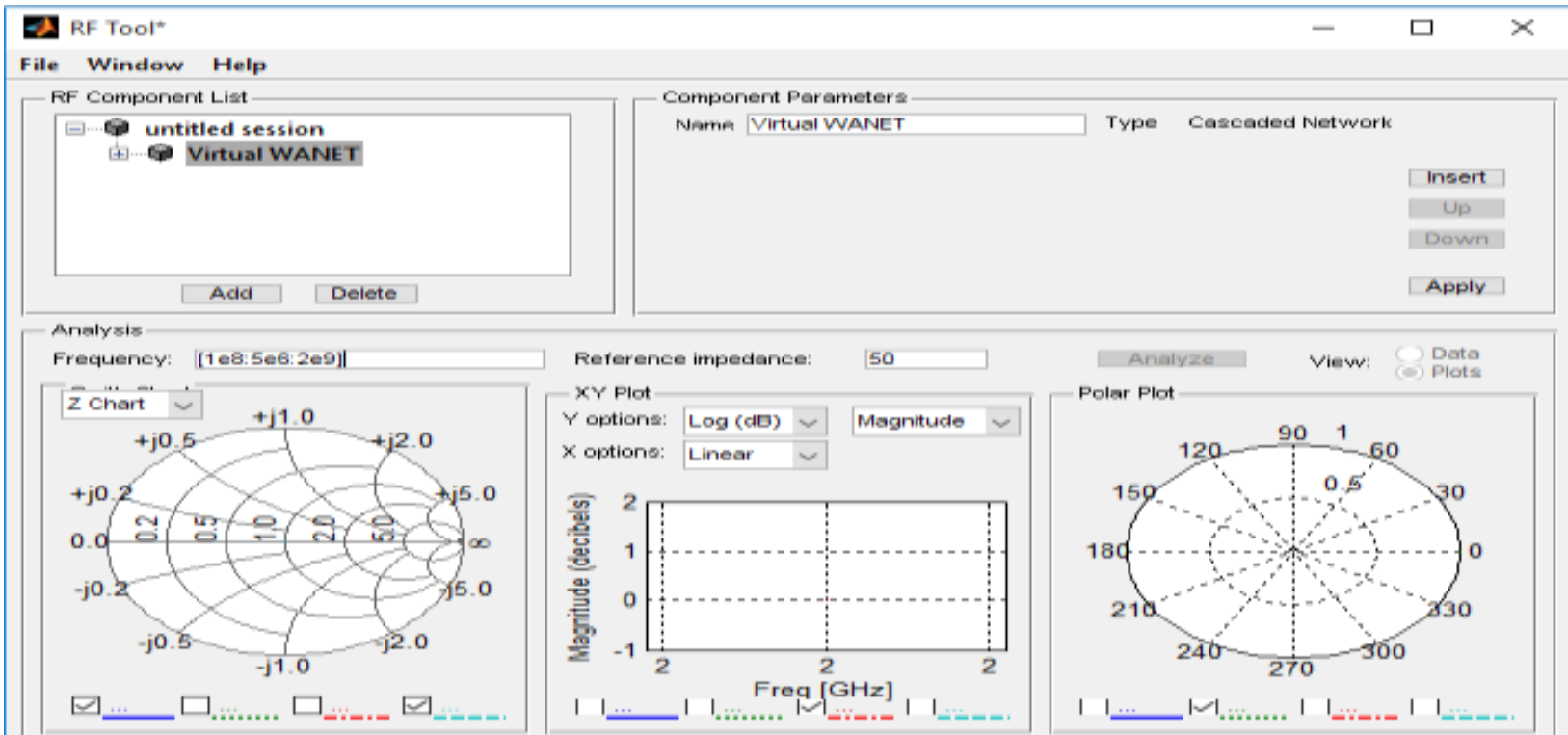

Fig. 4: MATLAB Radio Frequency Plot for Magnitude and Impedance

Filter Visualization Tool - Figure 1: Round-off Noise Power Spectrum

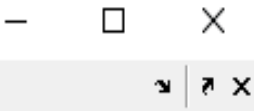

File Edit Analysis Insert View Debug Desktop Window Help

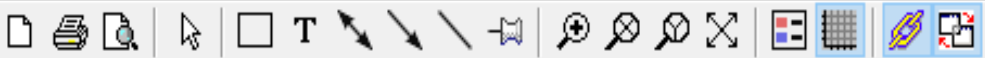

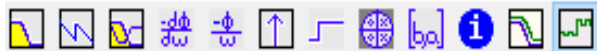

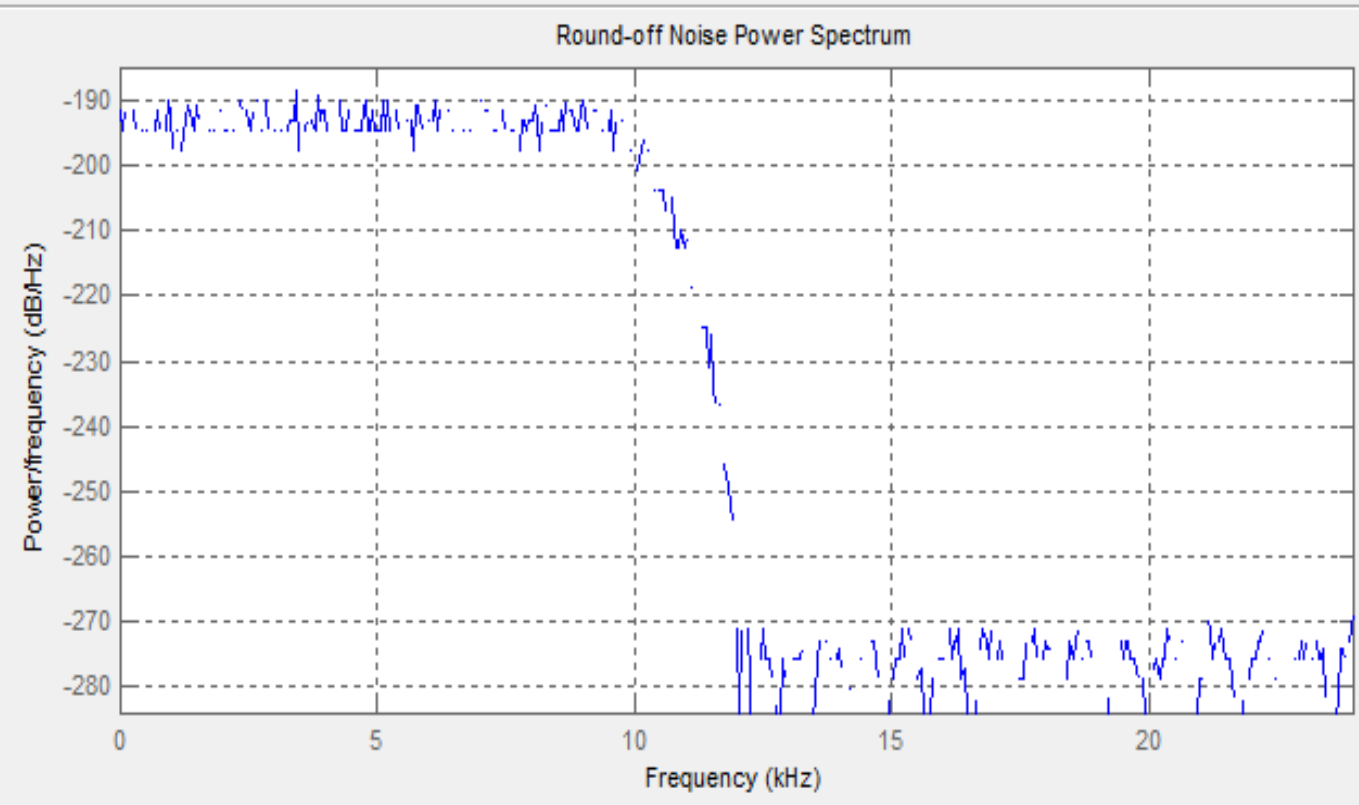

Fig. 5: MATLAB RF Plot for Channel Noise Suppression 


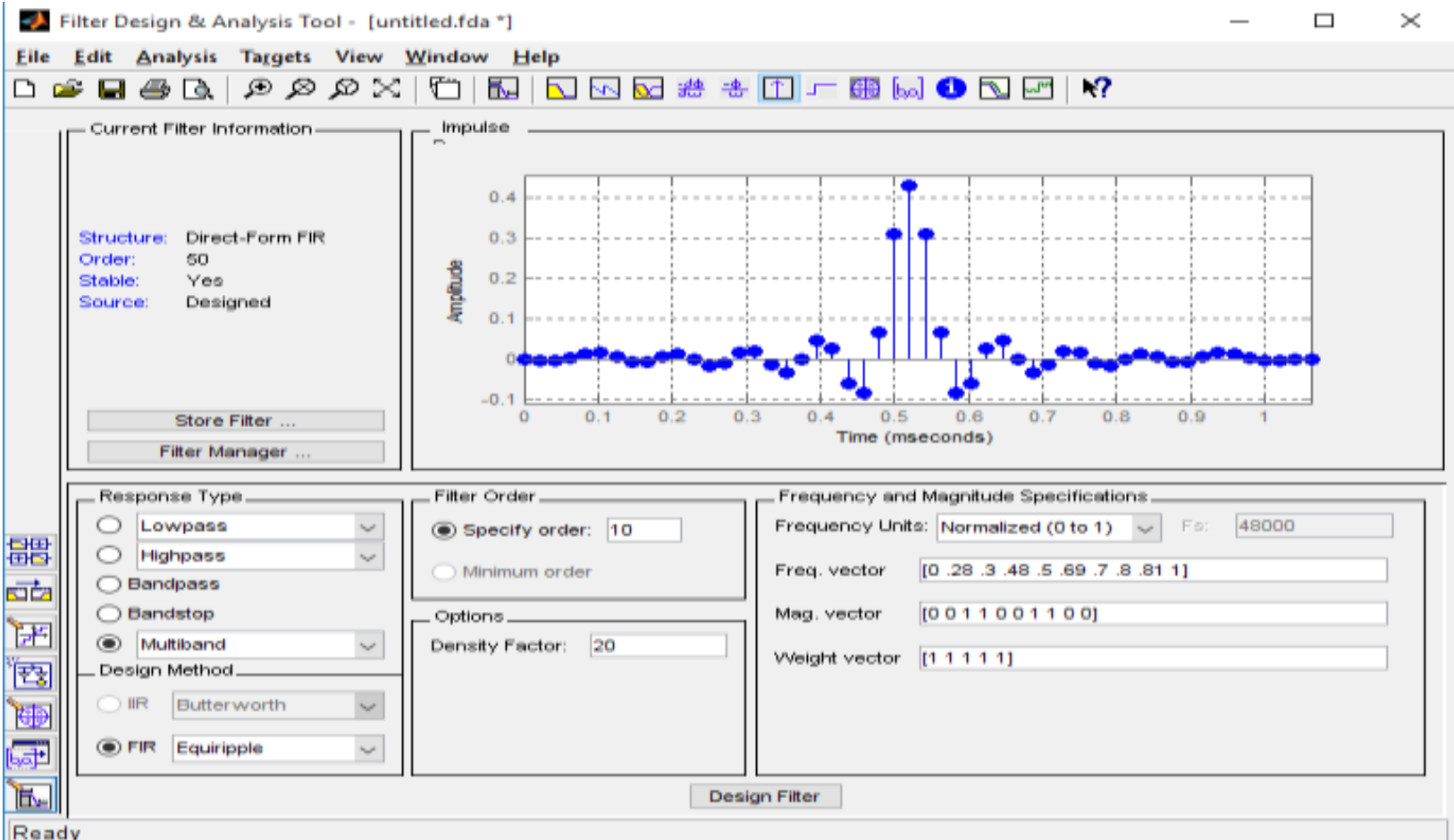

Fig. 6: MATLAB Time Interval for Frequency Impulse / Magnitude

\section{CONCLUSION}

Antennas are made intelligent by modifying the physical design to incorporate more elements and to transfer signals before transmission at every successive array. Signal gain is obtained by combining inputs from multiple antennas to optimize required power for establishing given level of coverage. Therefore, signal quality can be ascertained for desired throughput, if the steady flow of amplitude is maintained for identifying, recognizing and tracking signals of different devices without channel disturbances that could emanate to obstruct the propagation pattern.

\section{REFERENCES}

1. OpenTech, Smart Antenna Systems and Wireless Communication Network. Retrieved from http://www.google.com on $3^{\text {rd }}$ September, 2019.

2. H. Xiaomeng, "Improving the Convergence and Stability of Congestion Control Algorithm". IEEE Xplore Digital Library, 2007, 7(1), 206-215.

3. P. Sunita and T. Zaheeruddin, A review and comparison of quality of service routing in wireless Ad Hoc networks. International Journal of Wireless and Mobile Networks $(I J W M N) .2011,3(1), 11-21$.

4. K.A. Odekunle, O.C. Akinyokun, B.K. Alese, Development of an expert system for message routing in a switched network environment,2012, 10(2), 1-12.

5. D. Muhammad and L. Cecil, A Software Radio Architecture for Smart Antennas. Canada: Spectrum Signal Processing Inc, 2018,.

6. J. Litva and T. Lo, Digital Beamforming in Wireless Communications, Boston, MA: Artech House Publishing, 2008. 
7. O.W.Onifade, O.A.Ojesanmi, T.O. Oyebisi, Better Quality of Service Management with Fuzzy Logic in Mobile Adhoc Network. African Journal of Computing \& ICTs (AJOCICT). 2013,6(1). 59-68.

8. A.Adepoju, Path Determination for Effective Routing in Wireless Ad Hoc Network using Firefly Algorithm. M.Sc Dissertation: Ajayi Crowther University, Nigeria, 2018.

9. M.S.Manshahia, A Firefly Based Energy Efficient Routing in Wireless Sensor Networks. African Journal of Computing \& ICTs.2015, 8( 4), 27-32.

10. K.D.Anupama, Integrating Firefly Algorithm in Artificial Neural Network models (ANN) for accurate software cost predictions. Journal of Software: Evolution and Process, 2016, 28(8), 665-688.

11. S. Shehu, A. Mohammed, A.Y. Tambuwal, A. Bello, "Investigating Quality of Service Performance of Mobile Broadband Networks at Sokoto State Tertiary Institutions". Proceedings of the $1^{\text {st }}$ International AIT conference, 2015 70-75.

12. O.E.Ojo, A.B. Ajobiewe, T.D. Ajayi, Dynamic Congestion Control Scheme for Video Streaming in Peer to Peer Network, Proceedings of $1^{\text {st }}$ International Applied Information Technology conference,2015 107-113.

13. M.A. Abdala and A.K. Al-Zuhairy, Integrating of Smart Antenna System in Mobile Ad Hoc Network. International Journal of Machine Learning and Computing, 2013, 3(4), 342-346.

14. B. Haller, Algorithms and VLSI Architecture for RLS-Based Time Reference Beamforming in Mobile Communications," Proceedings of the International Zurich Seminar on Broadband Communications, Switzerland, 2008, 98-110.

15. V. Inzillo, F. De Rango, F. Zampogna, A.A. Quintana, . Smart Antenna Systems Model Simulation Design for 5G Wireless Network Systems.2018, DOI: 10.5772/intechopen.79933

\section{* Corresponding Author: Ayeni, A. Gabriel and Kabari, L. Giok;}

Post Graduate Unit (Ph.D), Department of Computer Science, Faculty of Natural and Applied Sciences, Post Graduate School, Rivers State University of Education, Port Harcourt, Nigeria 